\title{
Integral Performance Criteria Based Analysis of Load Frequency Control in Bilateral Based Market
}

\author{
P. Anitha' ${ }^{1}$ P. Subburaj ${ }^{2}$ \\ ${ }^{1}$ Department of Electrical Engineering, UVOCCE, Thoothukudi, India \\ ${ }^{2}$ Department of Electrical Engineering, NEC, Kovilpatti, India \\ Email: sakthistructrichy@gmail.com
}

Received 23 March 2016; accepted 24 May 2016; published 27 May 2016

Copyright (C) 2016 by authors and Scientific Research Publishing Inc.

This work is licensed under the Creative Commons Attribution International License (CC BY). http://creativecommons.org/licenses/by/4.0/

c) (i) Open Access

\begin{abstract}
Performance index based analysis is made to examine and highlight the effective application of Particle Swarm Optimization (PSO) to optimize the Proportional Integral gains for Load Frequency Control (LFC) in a restructured power system that operates under Bilateral based policy scheme. Various Integral Performance Criteria measures are taken as fitness function in PSO and are compared using overshoot, settling time and frequency and tie-line power deviation following a step load perturbation (SLP). The motivation for using different fitness technique in PSO is to show the behavior of the controller for a wide range of system parameters and load changes. Error based analysis with parametric uncertainties and load changes are tested on a two-area restructured power system. The results of the proposed PSO based controller show the better performance compared to the classical Ziegler-Nichols (Z-N) tuned PI and Fuzzy Rule based PI controller.
\end{abstract}

\section{Keywords}

Load Frequency Control, Particle Swarm Optimization, Bilateral Market, Area Control Error, Fuzzy Rule Based PI Controller, Parametric Uncertainties

\section{Introduction}

In general LFC role is to ensure reliable operation of interconnected power systems by adjusting generation to minimize frequency deviations and regulate tie-line flows. Operating the power systems in a new environment will certainly be more complex than in the past, due to restructuring and a considerable degree of technical and economical interconnections. LFC and relative control strategies mostly remained similar to before deregulation 
except that services provided by participants are now classified as ancillary [1]. For satisfactory operation of power units running in parallel, it is most desirable to have the frequency and tie-line power fixed on their nominal and scheduled values even when the load alters and, therefore, to remove ACE.

The LFC issue as an ancillary service represents an important role to maintain an acceptable level of efficiency, quality, and reliability in a deregulated power system environment. Possible issues in Frequency control in energy market were discussed in [1]. Vertically integrated structure, Bilateral based scheme modeling was discussed in [2] [3]. Many researchers have started to analyze possible new LFC schemes and regulation solutions, with paradigms suited for the energy market scenarios [4]-[9]. Fuzzy based intelligent controller was adapted and discussed in [6] [7]. The objective of the proposed controller in bilateral market is to reduce damping of frequency and tie-line power deviations oscillation for all admissible uncertainties and load changes. So, three different testing conditions are taken to test the robustness of the controllers.

This paper is organized as follows: Generalized bilateral scheme is presented in Section 2 and GPM is defined in this section. System taken for investigation is defined in Section 3. Fuzzy rule based control scheme for a bilateral based restructured power system is presented in Section 4. PSO based controller is discussed in Section 5 and the motivation of using different fitness function is discussed in this section. The objective of this work is to formulate the dynamic LFC and evaluate and analyze the LFC based on different performance criteria under parametric uncertainties.

\section{Generalized Bilateral Scheme}

New market concepts were adapted to achieve the goal of better performance and efficiency in restructured power systems. In this paper, bilateral based market is taken for analysis. A general configuration for the LFC [2] [3] in a deregulated environment is shown in Figure 1.

Bilateral based deregulated environment consist of an Independent System Operator (ISO), Distribution Companies (DISCOs), Generation Companies (GENCOs), and Transmission Companies (TRANSCOs). In the new environment, DISCOs may contract power from any GENCOs and ISO have to supervise these contracts [9]. The "Generation Participation Matrix (GPM)" shows the participation factor of each Genco in the considered control areas, and each control area is determined by a Disco. The rows of a GPM correspond to Gencos and the columns to the control areas that contract power. For example, the GPM for a large scale power system with $m$ control areas (Discos) and $n$ Gencos, has the following structure, in which gpfij refers to "generation participation factor" and shows the participation factor of Genco $i$ in total load following the requirement of DISCO $j$ based on a specified bilateral contract [10] in Equation (1).

New information signals due to possible various contracts between Disco i and other Discos and Gencos are shown as dashed arrows in Figure 1.

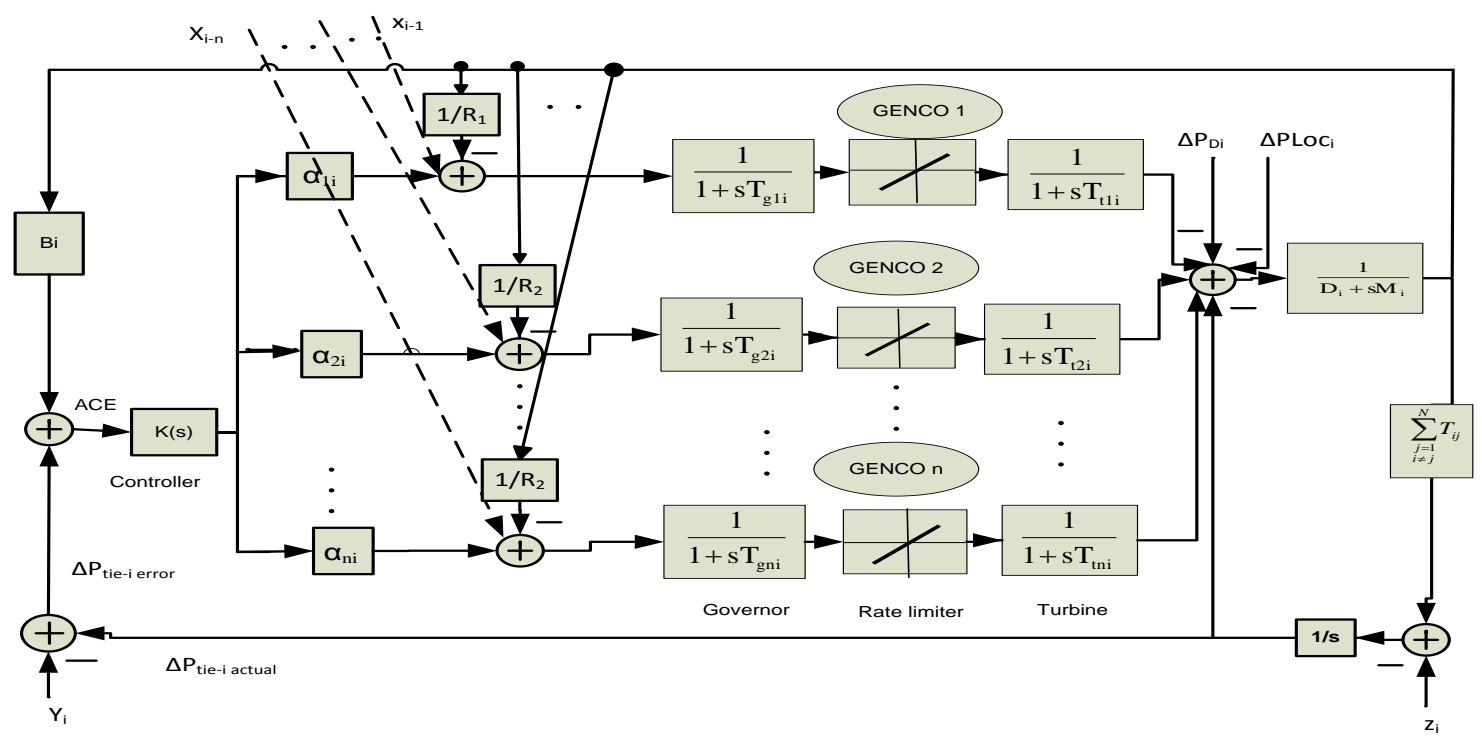

Figure 1. Bilateral based LFC model. 


$$
\begin{gathered}
G P M=\left[\begin{array}{ccccc}
g p f_{11} & g p f_{12} & \cdots & g p f_{1(m-1)} & g p f_{1 m} \\
g p f_{21} & g p f_{22} & \cdots & g p f_{2(m-1)} & g p f_{2 m} \\
\vdots & \vdots & \vdots & \vdots & \vdots \\
g p f_{(n-1) 1} & g p f_{(n-1) 2} & \cdots & g p f_{(n-1)(m-1)} & g p f_{(n-1) m} \\
g p f_{n 1} & g p f_{n 2} & \cdots & g p f_{n(m-1)} & g p f_{n m}
\end{array}\right] \\
\Delta P_{\text {tie-i,error }}=\Delta P_{\text {tie-i,actual }}-\sum(\text { Total export power - Total import Power }) \\
=\Delta P_{\text {tie-i,actual }}-\sum_{\substack{j=1 \\
j \neq i}}^{N}\left(\sum_{k=1}^{n} g p f_{k j}\right) \Delta P_{L j}-\sum_{k=1}^{n}\left(\sum_{\substack{j=1 \\
j \neq i}}^{N} g p f_{k j}\right) \Delta P_{L i}
\end{gathered}
$$

where

$$
\begin{gathered}
\Delta P_{\text {tie-i,scheduled }}=\sum_{\substack{j=1 \\
j \neq i}}^{N}\left(\sum_{k=1}^{n} g p f_{k j}\right) \Delta P_{L j}-\sum_{k=1}^{n}\left(\sum_{\substack{j=1 \\
j \neq i}}^{N} g p f_{k j}\right) \Delta P_{L i} \\
\sum_{i=1}^{n} g p f_{i j}=1 \\
\sum_{k=1}^{n} \alpha_{k i}=1 ; 0 \leq \alpha_{k i} \leq 1 \\
\Delta P_{m i}=\sum_{j=1}^{N} g p f_{i j} \Delta P_{L j}
\end{gathered}
$$

where $\Delta P_{d i}$ (Figure 1) is the area load disturbance, $\Delta P_{\text {Loc- } i}$ is the contracted load demand (contracted and uncontracted) in area $i$, and $\Delta P_{\text {tie-l,actual }}$ is the actual tie-line power in area $i$. Using Equation (2), the scheduled tie-line power $\left(\Delta P_{\text {tie-I, scheduled }}\right)$ can be calculated using Equation (7).

In the bilateral LFC structure, Control is highly decentralized. Each Load Matching Contract requires a separate control process, yet these control processes must cooperatively interact to maintain system frequency and minimize the area control error [4], where

$B_{i}$ frequency bias,

$\Delta P_{\text {tie_i } i}$ net tie-line power flow,

$\Delta P_{d i}$ area load disturbance between areas $i$ and $j$,

$\alpha$, area control error (ACE) participation factor,

$\Delta P_{L i}$ contracted demand of area $i$,

$\Delta P_{m i}$ power generation of Genco $i$,

$\Delta P_{\text {Loc } \_i}$ total local demand (contracted and uncontracted) in area $i$,

$\Delta P_{\text {tie_i, actual }}$ actual $\Delta P_{\text {tie_i }}$.

\section{System Modeling}

Consider the load frequency control problem for a two-area power system as shown in Figure 2. Each control area has the structure as shown in Figure 1. The control area considered here is a simplified power system consisting of two generating units. According to [11], the collective performance of all generators in the system is the interesting part in the analysis of LFC. Genco's considered here are reheat thermal units. The inter-machine oscillations are not considered. It is assumed that the response of all generators to changes in system load are coherent and can be represented by an equivalent generator, which has an inertia constant and a damping constant equal to the sum of the inertia constants and damping constants of all the generating units. The model is simple but captures the essential dynamics of a power system and has been widely used for LFC design purpose. The load frequency control problem for a multi-area power system requires that not only the frequency deviation 


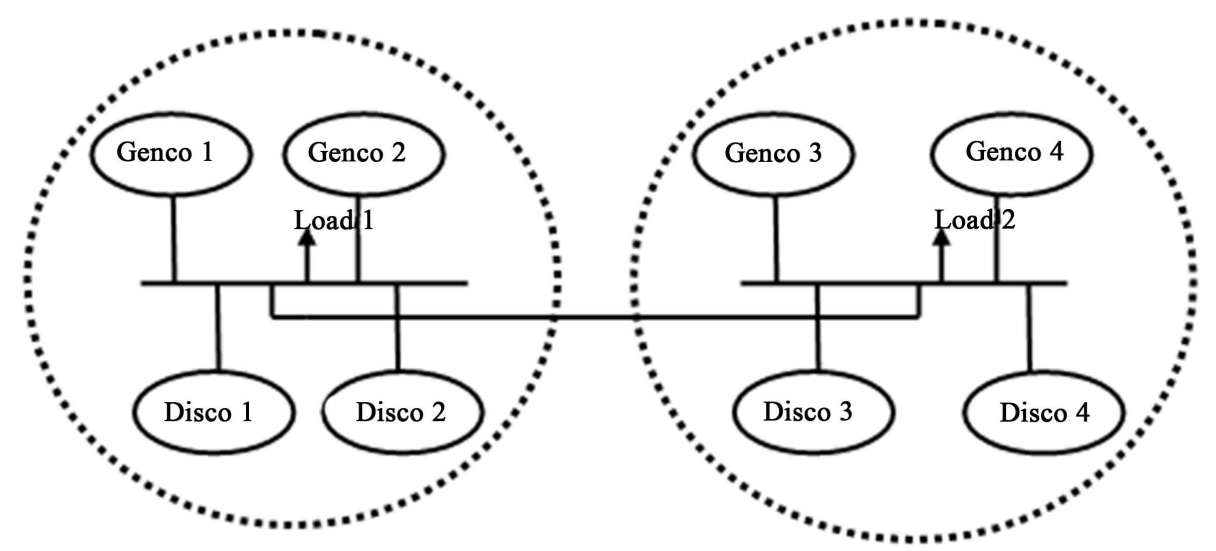

Figure 2. Two control area.

of each area must return to its nominal value but also the tie-line power flows must return to their scheduled values. So a composite variable, the area control error (ACE), is used as the feedback variable to ensure the two objectives.

For Area \#i, the area control error is defined as

$$
A C E_{i}=\Delta P_{\text {tie-i }}+B_{i} \Delta f_{i}
$$

and the feedback control for Area \#i take the form

$$
u_{i}=-k_{p i}(s) A C E_{i}-k_{I i}(s) \int A C E_{i}
$$

The transfer functions of the governor, the turbine, and the rotor inertia and load for Area \#i are denoted by $G_{g i}(s), G_{t i}(s)$, and $G_{p i}(s)$, respectively. The transfer functions of the above mentioned are represented as

$$
\begin{gathered}
G_{g i}(s)=\frac{1}{1+s T_{g i}} \\
G_{t i}(s)=\frac{1}{1+s T_{t i}} \\
G_{p i}(s)=\frac{K_{p i}}{1+s T_{p i}}
\end{gathered}
$$

then the transfer function from $u_{i}$ to $\Delta f_{i}$ can be easily found as

$$
G_{i}(s)=\frac{G_{p i} G_{t i} G_{g i}}{1+G_{p i} G_{t i} G_{g i} / R_{i}}
$$

The transfer function for Area \#i.

$$
P_{i}(s)=G_{i}(s) B_{i}
$$

The system parameters taken for investigation are given [12] in Appendix A.

\section{Fuzzy Rule Based Pi Controller (Fpi)}

Conventional control methods may not give satisfactory solutions, because of increasing complexity and changing structure of power systems. Fuzzy logic control is an excellent alternative to the conventional control methodology when the processes are too complex for analysis by conventional mathematical techniques. Fuzzy Logic Control consists of three main stages, namely the Fuzzification interface, the Inference rules engine and Defuzzification interface.

For LFC, ACE and its derivative are chosen as inputs and Proportional and Integral Gains are taken as control outputs of the fuzzy controller. The Membership Functions (MFs) were chosen to be triangular for obtaining fast 
response from the system. The MFs were named LN (Large Negative), MN (Medium Negative), SN (Small Negative), Z (Zero), SP (Small Positive), MP (Medium Positive) and LP (Large Positive). In [13], Ziegler-Nichols proposed a heuristic method to quickly adjust the controller parameters P, PI, PID. The MFs ranges of the inputs (Ace \& dAce) and control outputs (KP \& Ki) for area i are determined based on the experiments with classical (Ziegler-Nichols Tuned) PI controller. These two input signals are used as rule-antecedent in the formation of rule base, and the control outputs are used to represent the contents of the rule-consequent in performing the rule base [7]. The fuzzy rule base is framed using the control rules that the system will operate. If Ace is significantly bigger than the set value and dAce is increased rapidly, then the output of the controller, ui, is to be big. Centroid method is used in defuzzification process. Therefore, the output of the system goes to the set value. Based on the experiments with classical controller, fuzzy rules are formed and the appropriate rules for Ki and Kp are given in Table 1.

\section{PSO Based PI Controller}

PSO is a novel population based meta heuristic, which utilize the swarm intelligence generated by the cooperation and competition between the particles in a swarm and has emerged as a useful tool for engineering optimazation. It has also been found to be robust in solving problems featuring nonlinearity, non-differentiability and high dimensionality.

In PSO, each particle is flown through the multidimensional search space, adjusting its position in search space according to its own experience or knowledge and that of neighboring particles. Therefore, a particle makes use of the best position encountered by itself and the best position of its neighbors to position itself toward an optimum solution. The effect is that particles fly toward the global minimum, while still searching a wide area around the best solution. The performance of each particle is measured according to a predefined fitness function which is related to the problem being solved [14]. The upper limit of the random value is a system parameter specified by the user. The larger the upper limit, the more the trajectory of the particles oscillates. Smaller values ensure smooth trajectories. This upper limit prevents particles from moving too rapidly from one region in search space to another. Vmax is usually initialized as a function of the range of the problem. The inertia weight controls the influence of previous velocities on the new velocity. Large inertia weights cause larger exploration of the search space, while smaller inertia weights focus the search on a smaller region [15]. In this proposed PSO, the inertia weight $(\mathrm{w})$ is started with a large value, which is decreased over time.

PSO starts with a population of random solutions "particles" in a D-dimension space. The ith particle is represented by $X_{i}=\left(x_{i 1}, x_{i 2}, \cdots, x_{i D}\right)$. Each particle keeps track of its coordinates in hyperspace, which are associated with the fittest solution it has achieved so far. The value of the fitness for particle i (pbest) is also stored as $P_{i}=\left(p_{i 1}, p_{i 2}, \cdots, p_{i D}\right)$. The global version of PSO keeps track of the overall best value (gbest), and its location, obtained thus far by any particle in the population. PSO consists of, at each step, changing the velocity of each particle toward its pbest and gbest according to Equation (15). The velocity of particle i is represented as $V_{i}=\left(v_{i 1}, v_{i 2}, \cdots, v_{i D}\right)$. Acceleration is weighed by a random term, with separate random numbers being generated for acceleration toward pbest and gbest. The position of the ith particle is then updated according to Equation (16), [16]. Figure 3 shows the flowchart of the proposed PSO algorithm.

Table 1. Rules for fuzzy rule based pi controller.

\begin{tabular}{cccccccc}
\hline Ace/dAce & LN & MN & SN & Z & SP & MP & LP \\
\hline LN & LP & LP & LP & MP & MP & SP & Z \\
MN & LP & MP & MP & MP & SP & Z & SN \\
SN & LP & MP & SP & SP & Z & SN & MN \\
Z & MP & MP & SP & Z & SN & MN & MN \\
SP & MP & SP & Z & SN & SN & MN & LN \\
MP & SP & Z & SN & MN & MN & MN & LN \\
LP & Z & SN & MN & MN & LN & LN & LN \\
\hline
\end{tabular}




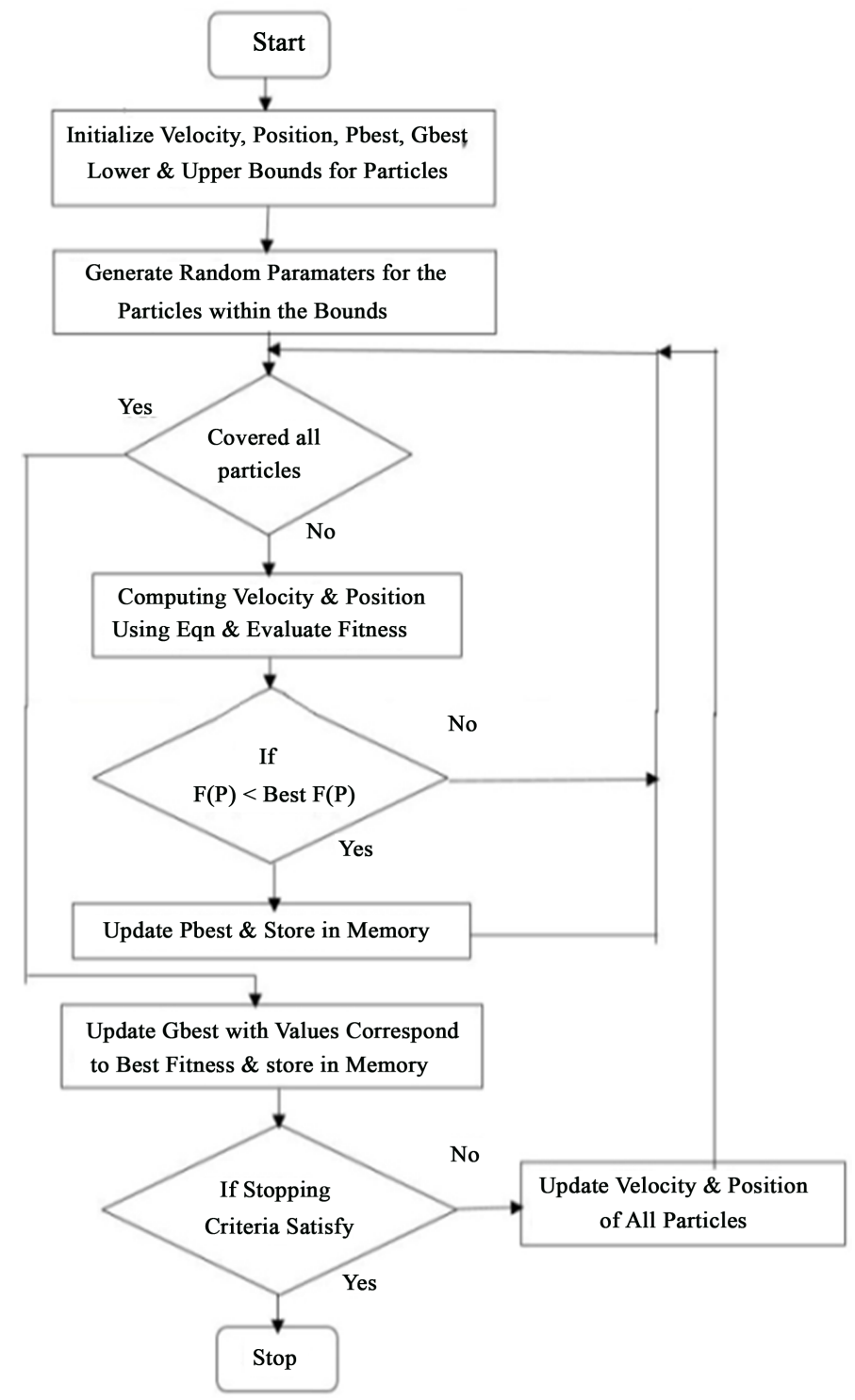

Figure 3. PSO flow chart.

$$
\begin{gathered}
v_{i D}=w \times v_{i D}+c 1 \times \operatorname{rand}() \times\left(p_{i d}-x_{i d}\right)+c 2 \times \operatorname{rand}() \times\left(p_{g d}-x_{i d}\right) \\
x_{i d}=x_{i d}+v_{i d}
\end{gathered}
$$

It should be noted that choice properly fitness function is very important in synthesis procedure. Because different fitness functions promote different PSO behaviors, which generate fitness value providing a performance measure of the problem considered [1]. Four kinds of performance criteria usually considered in the control design are the Integral of Time multiplied Absolute Error (ITAE), Integral of Squared Error (ISE), Integral of Time multiplied Squared Error (ITSE) and Integral of Absolute Error (IAE). ISE and ITAE criterions are often used in literature [7] [8] for their better performance compared to IAE and ITSE criterion. ISE criterion integrates the square of the error over time. ISE will penalize large errors more than smaller ones (since the square of a large error will be much bigger). ITAE criterion integrates the absolute value of the error over time. ITAE will penalize long duration transients and errors occurring later in the responses Similarly MSE have the potential for producing reductions in oscillations and tracking errors. For our optimization problem, the following fitness functions are introduced: 


$$
\begin{gathered}
f(I S E)=\int_{0}^{T}\left(\Delta P_{\text {tie- }-i}+B_{i} \Delta f\right)^{2} \mathrm{~d} T \\
f(I S E)=\int_{0}^{T}\left(\Delta P_{\text {tie- }-i}+B_{i} \Delta f\right)^{2} \mathrm{~d} T \\
f(I A E)=\int_{0}^{T}\left|\left(\Delta P_{\text {tie-i }}+B_{i} \Delta f\right)\right| d T \\
f(I T A E)=\int_{0}^{T} T\left|\left(\Delta P_{\text {tie-i }}+B_{i} \Delta f\right)\right| \mathrm{d} T \\
f(M S E)=\sum\left(\Delta P_{\text {tie-i }}(t)+B_{i} \Delta f(t)\right)^{2}
\end{gathered}
$$

The optimization problem is based on the minimization of the Fitness Function subject to the conditions that the PI gains $k_{p}$ and $k_{i}$ of both the controllers will lie within the minimum and the maximum limits as given below.

$$
k_{\text {PMin }} \leq k_{P} \leq k_{\text {PMax }} ; k_{\text {IMin }} \leq k_{I} \leq k_{\text {IMax }}
$$

\section{Simulation Results}

In order to illustrate the behavior of the proposed control strategy some simulations has been carried out. The system parameter of the test system is given in Appendix A.

Consider a system where all GENCOs in each area participate in LFC, i.e. ACE participation factors are $\alpha_{1}=$ $0.75, \alpha_{2}=1-\alpha_{1}=0.25 ; \alpha_{3}=0.5, \alpha_{4}=1-\alpha_{3}=0.5$

$$
G P M=\left|\begin{array}{cccc}
0.5 & 0.25 & 0 & 0.3 \\
0.2 & 0.25 & 0 & 0 \\
0 & 0.25 & 1 & 0.7 \\
0.3 & 0.25 & 0 & 0
\end{array}\right|
$$

Case I: In this case it is assumed that all Gencos are participating in the LFC task as per contract. It is assumed that a large step load 0.05 p.u MW is demanded by each DISCOs in all areas. Frequency response for both areas and Power generation of Gencos are shown in Figures 4-6. It is observed that PSO based controller has low overshoot and less settling time compared to Fuzzy Rule based PI(FPI) Controller. It is noted that PSO-ITAE and PSO-ITSE has better performance than PSO-IAE, PSO-ISE and PSO-MSE. The actual power generated by each Gencos reach the desired value is given in Table 2.

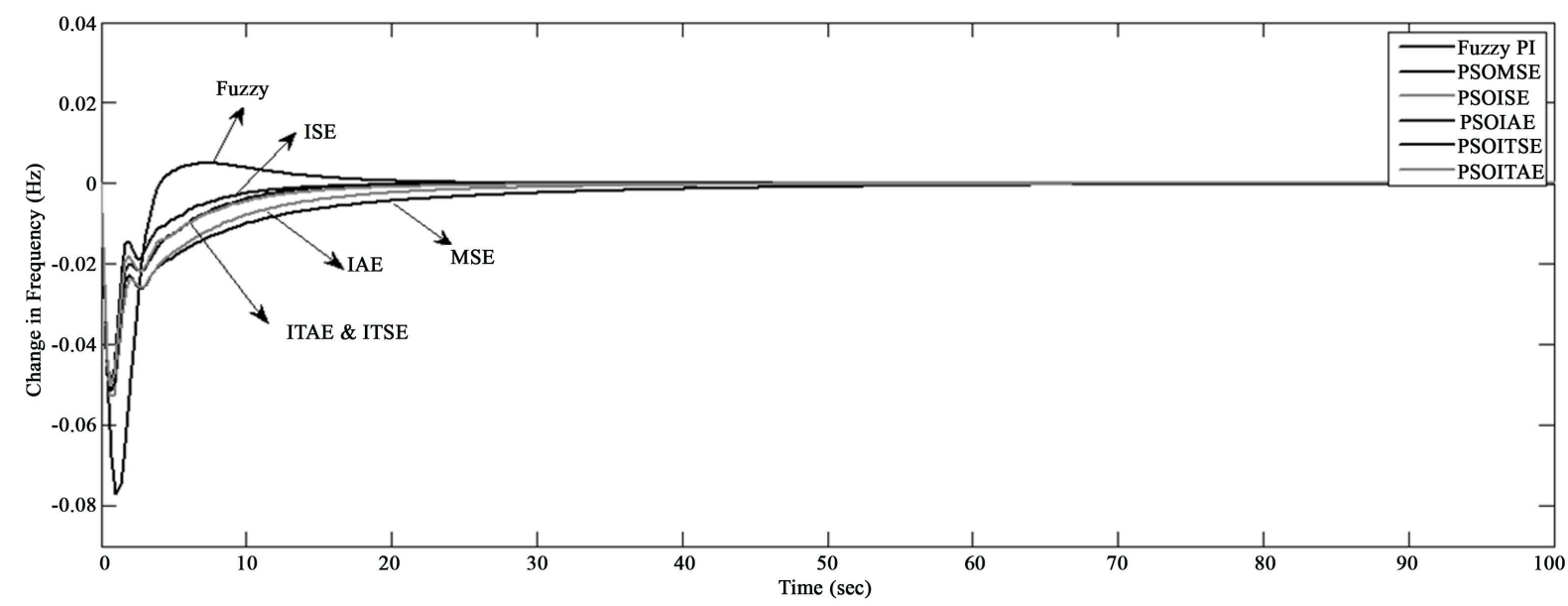

Figure 4. Frequency deviation for Area-1 Case-I. 


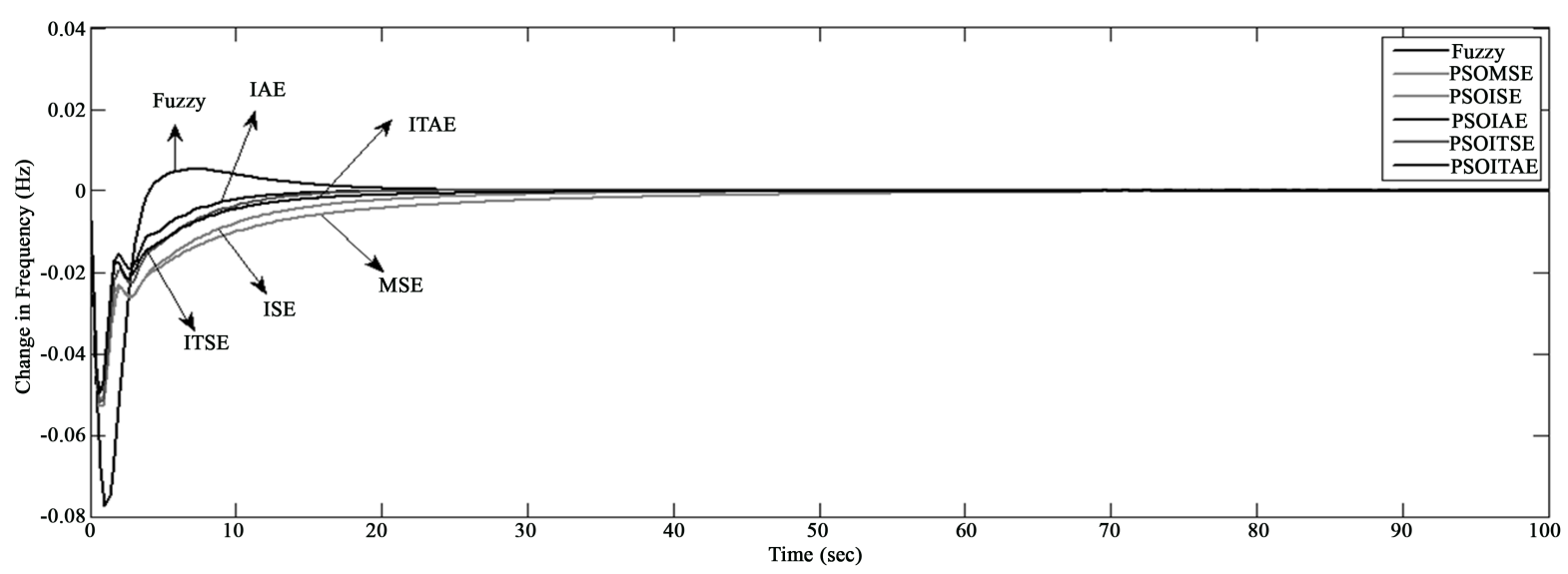

Figure 5. Frequency deviation for Area-2 Case-I.

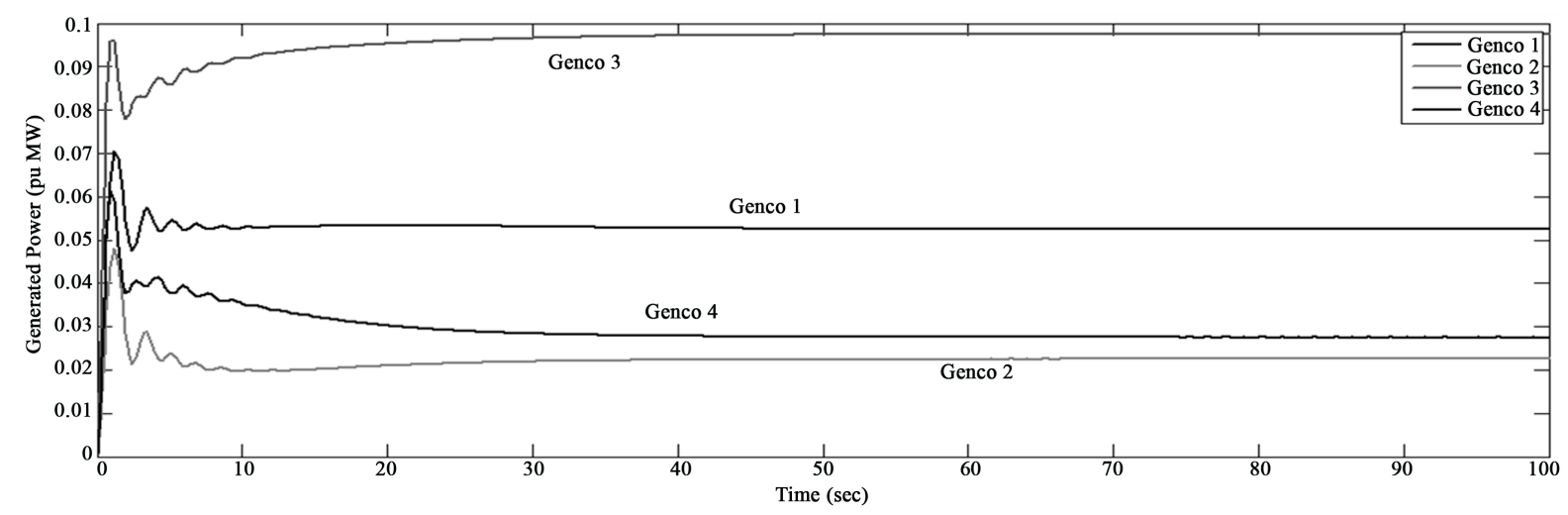

Figure 6. Power generated by GENCO’S Case I.

Table 2. Generated power in response to case 1.

\begin{tabular}{ccccc}
\hline Genco & 1 & 2 & 3 & 4 \\
\hline$\Delta \mathrm{P}_{\mathrm{mi}}($ puMW) & 0.0525 & 0.0225 & 0.0975 & 0.0275 \\
\hline
\end{tabular}

Case II: It is assumed that the rotating mass and load pattern parameters Di and Mi have uncertain values in each control area. In addition to case I, 25\% decrease in parametric uncertainties is taken. The chane in frequency deviation for both areas are shown in Figure 7 and Figure 8. Simulation results show that all the PSObased PI controllers track the load fluctuations possibly good. In particular PSO-ISE \&PSO-ITSE are superior to other methods. Their overshoot \& settling time are better than other controllers. The performance measures like overshoot and settling time are compared in Table 3.

Case III: Consider Case II, in addition to the specified contracted load demand and 25\% decresase in $\mathrm{D}_{\mathrm{i}}$ and $\mathrm{M}_{\mathrm{i}}$, a bounded random step load change $\left(\Delta P_{d i}\right)$ as a uncontracted demand appears in each control area.

$$
(-0.05 \mathrm{pu}) \leq \Delta P_{d i} \leq(+0.05 \mathrm{pu})
$$

The main aim of this test is to check the robustness of the proposed controller against uncertainties and random load disturbance. Frequency deviation for two areas are given in Figure 9 and Figure 10. Random load pattern applied to area1 and area 2 are given in Figure 11 and Figure 12. The results shows that PSO controller quickly find its optimum value compared to fuzzy controller.

The optimum values of the Proportional and Integral Gains of the different controllers for the aforementioned cases are shown in Table 4. 


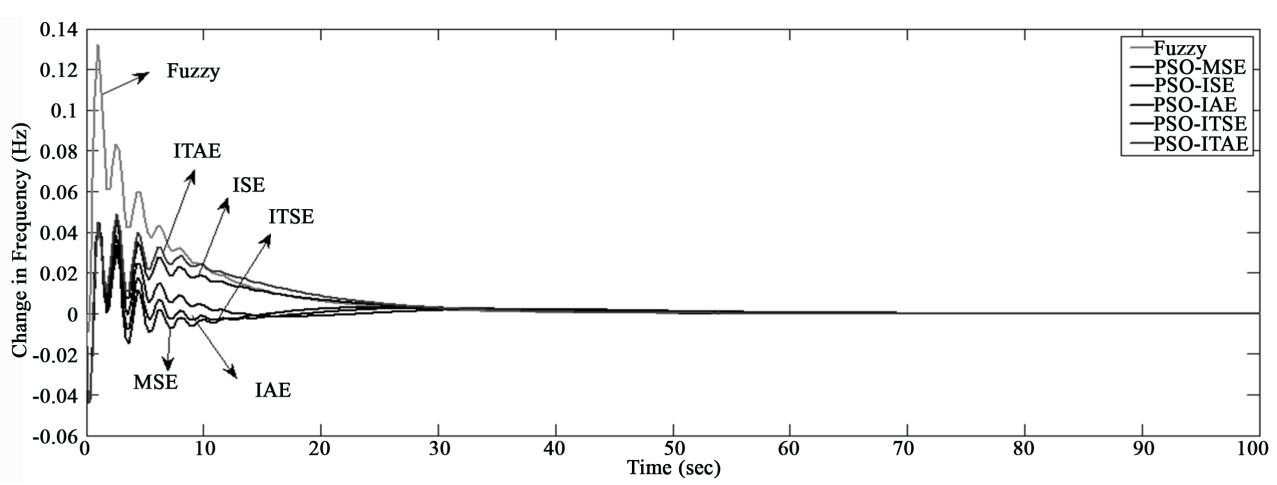

Figure 7. Frequency deviation for Area-1 Case-II.

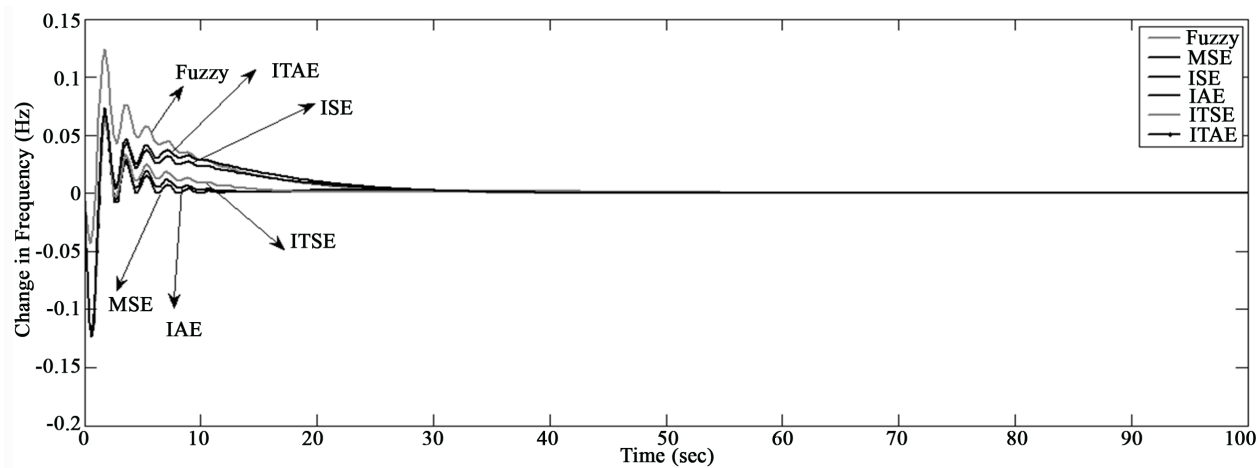

Figure 8. Frequency deviation for Area-2 Case-II.

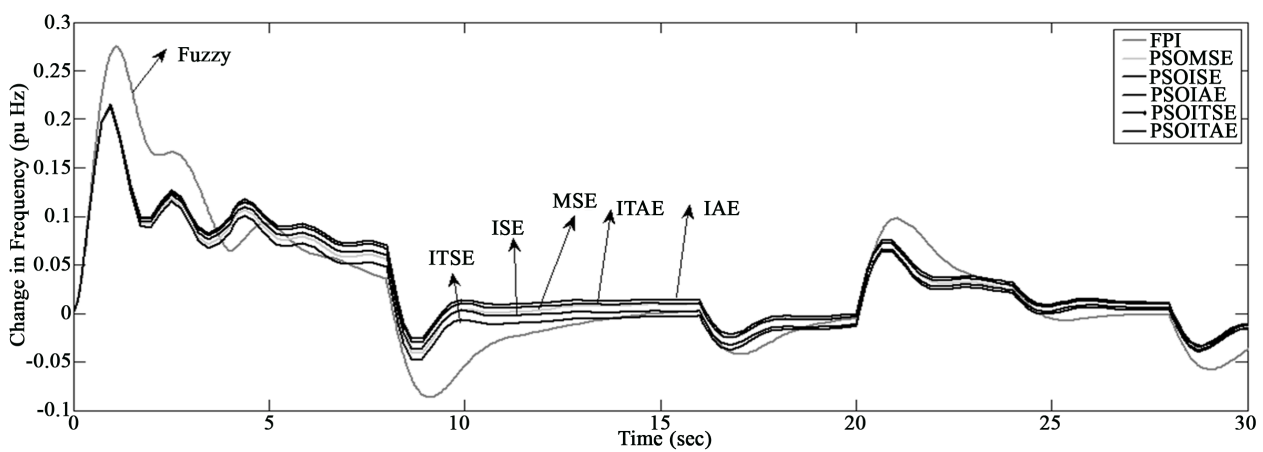

Figure 9. Frequency deviation for Area-1 Case III.

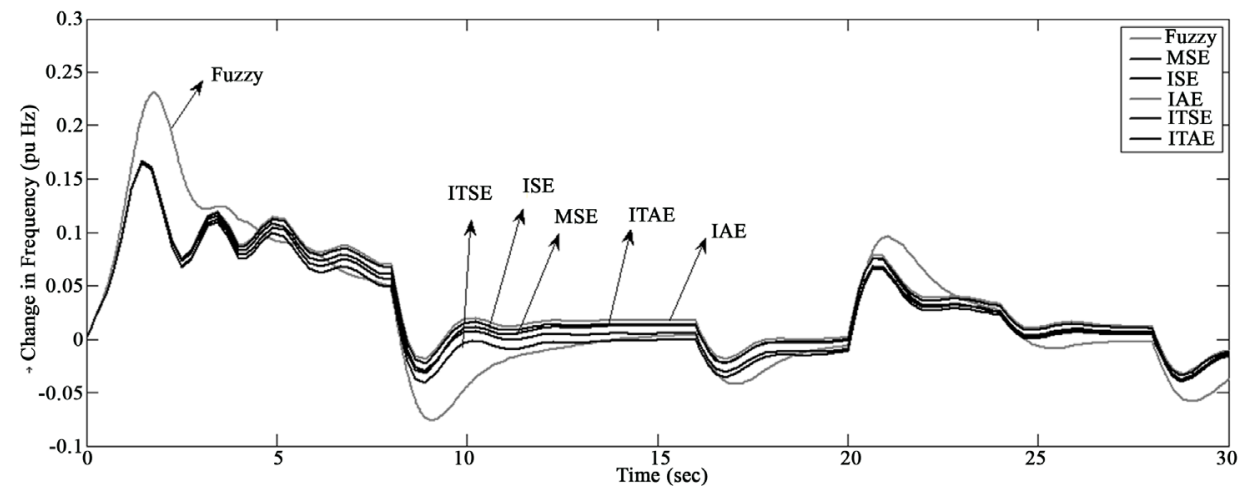

Figure 10. Frequency deviation for Area 2 Case III. 


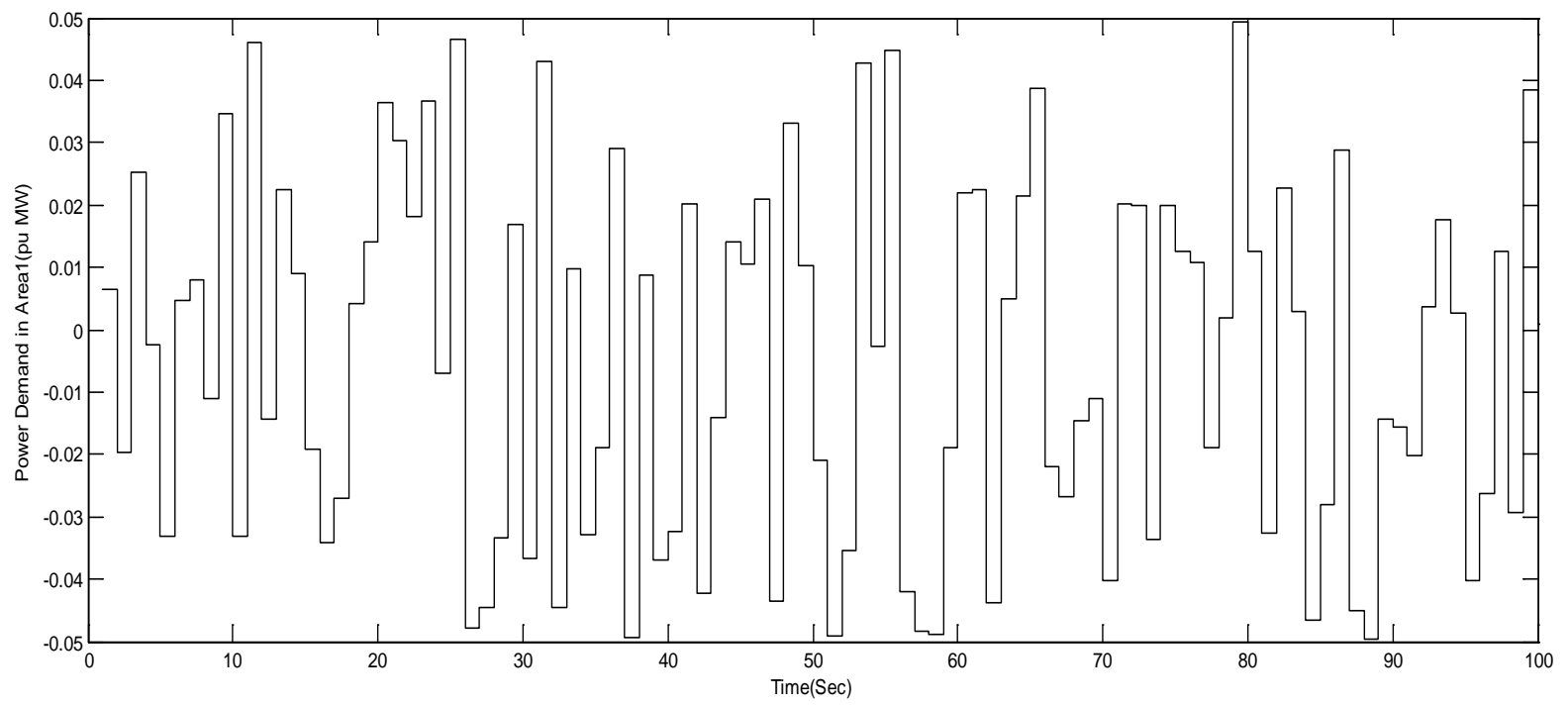

Figure 11. Random disturbance load pattern in Area 1.

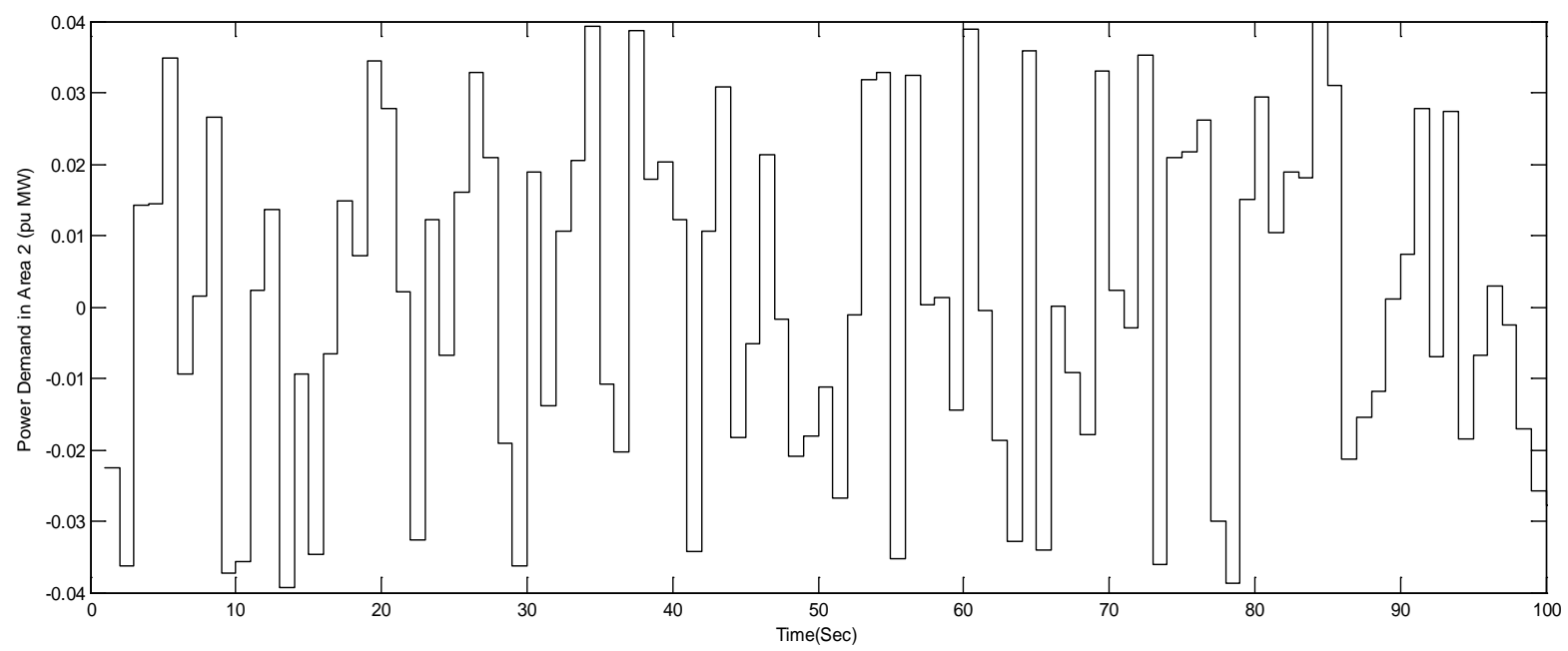

Figure 12. Random disturbance load pattern in Area 2.

Table 3. Performance measure for Case 2.

\begin{tabular}{ccccc}
\hline \multirow{2}{*}{ Controller } & \multicolumn{2}{c}{ Area1 } & \multicolumn{2}{c}{ Area2 } \\
\cline { 2 - 5 } & Overshoot (Hz) & Settling Time (Sec) & Overshoot (Hz) & Settling Time (Sec) \\
\hline Classical Z-N(Z-N) & +0.162 & 66 & +0.141 & 62 \\
Fuzzy PI(FPI) & +0.126 & 43 & +0.120 & 45 \\
PSO-MSE & +0.045 & 52 & -0.125 & 40 \\
PSO-ISE & +0.046 & 34 & -0.115 & 32 \\
PSO-IAE & +0.045 & 49 & -0.115 & 29 \\
PSO-ITSE & +0.042 & 34 & -0.110 & 35 \\
PSO-ITAE & +0.05 & 34 & -0.115 & 29 \\
\hline
\end{tabular}


Table 4. Optimal value of controller parameter for Area 1 and Area 2.

\begin{tabular}{|c|c|c|c|c|c|c|c|c|c|c|c|c|}
\hline \multirow{3}{*}{ Controller } & \multicolumn{6}{|c|}{ Area 1} & \multicolumn{6}{|c|}{ Area 2} \\
\hline & \multicolumn{2}{|c|}{ Case 1} & \multicolumn{2}{|c|}{ Case 2} & \multicolumn{2}{|c|}{ Case 3} & \multicolumn{2}{|c|}{ Case 1} & \multicolumn{2}{|c|}{ Case 2} & \multicolumn{2}{|c|}{ Case 3} \\
\hline & $\mathrm{K}_{\mathrm{P} 1}$ & $\mathrm{~K}_{\mathrm{I} 1}$ & $\mathrm{~K}_{\mathrm{P} 1}$ & $\mathrm{~K}_{\mathrm{I} 1}$ & $\mathrm{~K}_{\mathrm{P} 1}$ & $\mathrm{~K}_{\mathrm{I} 1}$ & $\mathrm{~K}_{\mathrm{P} 2}$ & $\mathrm{~K}_{\mathrm{I} 2}$ & $\mathrm{~K}_{\mathrm{P} 2}$ & $\mathrm{~K}_{\mathrm{I} 2}$ & $\mathrm{~K}_{\mathrm{P} 2}$ & $\mathrm{~K}_{\mathrm{I} 2}$ \\
\hline $\mathrm{Z}-\mathrm{N}$ & 0.2405 & 0.715 & 0.197 & 0.605 & 0.195 & 0.625 & 0.345 & 0.745 & 0.3210 & 0.7127 & 0.3205 & 0.5421 \\
\hline FPI & 0.175 & 0.250 & 0.20 & 0.555 & 0.210 & 0.545 & 0.2331 & 0.3701 & 0.2051 & 0.685 & 0.2123 & 0.680 \\
\hline PSO-ISE & 0.325 & 0.4205 & 0.301 & 0.4265 & 0.3250 & 0.4135 & 0.2505 & 0.5214 & 0.2765 & 0.4157 & 0.2706 & 0.4710 \\
\hline PSO-ITSE & 0.3015 & 0.4175 & 0.3105 & 0.4956 & 0.320 & 0.3915 & 0.2831 & 0.5001 & 0.2871 & 0.4101 & 0.2801 & 0.4611 \\
\hline PSO-IAE & 0.2971 & 0.4103 & 0.310 & 0.7101 & 0.3221 & 0.4001 & 0.2768 & 0.510 & 0.2715 & 0.4423 & 0.2900 & 0.4733 \\
\hline PSO-ITAE & 0.3197 & 0.4123 & 0.3321 & 0.5191 & 0.3210 & 0.4200 & 0.2899 & 0.5021 & 0.2665 & 0.4741 & 0.2899 & 0.4777 \\
\hline PSO-MSE & 0.4550 & 1.012 & 0.3001 & 0.9123 & 0.4000 & 0.920 & 0.9245 & 1.3232 & 0.7001 & 1.0219 & 0.6748 & 1.0114 \\
\hline
\end{tabular}

\section{Conclusion}

In this paper, PSO based PI tuning are applied to bilateral LFC scheme. Simulation results indicate the effectiveness of the proposed controller in damping the frequency oscillations and tie-line power very fast with less undershoot and overshoot. It is also seen from simulation results that under normal circumstances, Fuzzy and all the PSO-based controllers produce the better results. During parameter uncertainties, the proposed controller with ISE penalizes large initial and final error. During large load disturbance, it penalizes excessively over damped oscillations and hence PSO-ISE consecutively produces optimum result in all testing conditions.

\section{References}

[1] Christie, R.D. and Bose, A. (1996) Load Frequency Control Issues in Power System Operations after Deregulation. IEEE Transactions on Power Systems, 11, 1191-1200. http://dx.doi.org/10.1109/59.535590

[2] Donde, V., Pai, M.A. and Hiskens, I.A. (2001) Simulation and Optimization in an AGC System after Deregulation. IEEE Transactions on Power Systems, 16, 481-489. http://dx.doi.org/10.1109/59.932285

[3] Bevrani, H., Mitani, Y., Tsuji, K. and Bevrani, H. (2005) Bilateral Based Robust Load Frequency Control. Energy Conversion and Management, 46, 1129-1146. http://dx.doi.org/10.1016/j.enconman.2004.06.024

[4] Delfino, B., Fornari, F. and Massucco. S. (2002) Load-Frequency Control and Inadvertent Interchange Evaluation in Restructured Power Systems. IEE Proceedings_Generation, Transmission and Distribution, 5, 607-614. http://dx.doi.org/10.1049/ip-gtd:20020368

[5] Bevrani, H., Mitani, Y. and Tsuji, K. (2004) Robust Decentralised AGC in Restructured Power System. Energy Conversion and Management, 45, 2297-2312. http://dx.doi.org/10.1016/j.enconman.2003.11.018

[6] Shayeghi, H., Shayanfar, H.A. and Jalili, A. (2006) Multi Stage Fuzzy PID Power System Automatic Generation Controller in the Deregulated Environment. Energy Conversion and Management, 47, 2829-2845. http://dx.doi.org/10.1016/j.enconman.2006.03.031

[7] Shayeghi, H., Jalili, A. and Shayanfar, H.A. (2007) Robust Modified GA Based Multi-Stage Fuzzy LFC. Energy Conversion and Management, 48, 1656-1670. http://dx.doi.org/10.1016/j.enconman.2006.11.010

[8] Daneshfar, F. (2013) Intelligent Load-Frequency Control in a Deregulated Environment: Continuous-Valued Input, Extended Classifier System Approach. IET Generation, Transmission \& Distribution, 7, 551-559. http://dx.doi.org/10.1049/iet-gtd.2012.0478

[9] Nanda, J., Mishra, S. and Saikia, L.C. (2009) Maiden Application of Bacterial Foraging Based Optimization Technique in Multi-Area Automatic Generation Control. IEEE Transactions on Power Systems, 24, 602-609. http://dx.doi.org/10.1109/TPWRS.2009.2016588

[10] Bevrani, H. and Hiyama, T. (2011) Intelligent Automatic Generation Control. CRC Press, 77-85.

[11] Kundur, P. (2008) Power System Stability and Control. Tata McGraw Hill, New Delhi, 581-626.

[12] Saadat and Hadi (2010) Power System Analysis. Tata McGraw-Hill, 542-555.

[13] Ziegler, J.G. and Nichols, N.B. (1942) Optimum Settings for Automatic Controllers. ASME, 64, 759-768. 
[14] Kennedy, J. and Eberhart, R. (1995) Particle Swarm Optimization. Proceedings of IEEE International Conference on Neural Networks, 4, 1942-1948. http://dx.doi.org/10.1109/icnn.1995.488968

[15] Clerc, M. and Kennedy, J. (2002) The Particle Swarm-Explosion, Stability, and Convergence in a Multidimensional Complex Space. IEEE Transactions on Evolutionary Computation, 6, 58-73. http://dx.doi.org/10.1109/4235.985692

[16] Kennedy, J., Eberhart, R. and Shi, Y. (2001) Swarm Intelligence. Morgan Kaufman Publishers, San Francisco.

\section{Appendix A}

$$
\begin{aligned}
& R_{1}=R_{3}=3 \% ; \\
& R_{2}=R_{4}=2.73 \% ; \\
& T_{g 1}=T_{g 3}=0.08 \mathrm{~s} ; \\
& T_{g 2}=T_{g 4}=0.06 \mathrm{~s} ; \\
& T_{t 1}=T_{t 3}=0.4 \mathrm{~s} ; \\
& T_{t 2}=T_{t 4}=0.44 \mathrm{~s} ; \\
& K_{r 1}=K_{r 2}=K_{r 3}=K_{r 4}=0.5 ; \\
& T_{r 1}=T_{r 2}=T_{r 3}=T_{r 4}=10 \mathrm{~s} ; \\
& D_{1}=0.015 \mathrm{pu} \mathrm{MW/Hz} ; \\
& D_{2}=0.016 \mathrm{pu} \mathrm{MW/Hz;} \\
& M_{1}=0.16667 ; \\
& M_{2}=0.2012 ; \\
& B_{1}=0.3483 ; \\
& B_{2}=0.3827 ; \\
& T_{12}=0.20
\end{aligned}
$$

